\title{
The use of a non-pedal tricycle to promote physical activity in nursing home residents: a pilot study
}

\author{
TATIANE CALVE ${ }^{1,2} \mid$ ANA M. F. BARELA² \\ ${ }^{1}$ Escola de Educação - Centro Universitário Internacional - UNIINTER, Curitiba, PR, Brasil. \\ 2 Instituto de Ciências da Atividade Física e Esporte, Universidade Cruzeiro do Sul, São Paulo, SP, Brasil. \\ Correspondence to: Profa. Dra. Ana Maria Forti Barela, Universidade Cruzeiro do Sul, Rua Galvão Bueno, 868, Liberdade, São Paulo, SP, Brazil, $01506-000$. \\ email: ambarela@gmail.com \\ https://doi.org/10.20338/bjmb.v15i2.194
}

\begin{abstract}
HIGHLIGHTS
- Non-pedal tricycle enables inactive elderlies to move around safely.

- The sessions with a non-pedal tricycle promoted improvement in walking resistance.

- The sessions with a non-pedal tricycle promoted improvement in walking speed.

- The use of non-pedal tricycle promotes benefits to elderlies living in nursing homes
\end{abstract}

ABBREVIATIONS

CG Control group

IG Intervention group

ANOVAs Analyses of variance
BACKGROUND: Elderly individuals living in nursing homes are usually inactive and do not seem motivated to engage in physical activity. Therefore, it is important to investigate new possibilities that enable them to be active in these environments.

AIM: To investigate the feasibility of a protocol using a non-pedal tricycle in elderly individuals living in nursing homes and its effects on improving endurance, walking speed, and functional mobility.

METHOD: Fourteen nursing home residents aged from 60 to 93 years were included in the study protocol. They were randomly allocated into two groups: control (CG) and intervention (IG). The CG kept their routine during the engagement in the study, and the IG, in addition to the routine, used a non-pedal tricycle to move around the nursing home twice a week for 12 weeks. Participants from both groups underwent the 6-minute walk, 10-meter walk, and timed-up and go tests, one week before the first practice session and one week after the last practice session provided to the IG.

RESULTS: Before the intervention, there was no group difference in any test, and after the intervention, only IG participants presented an increase in walking distance and walking speed. Both groups did not change their time to conclude the timed-up and go test.

CONCLUSION: The use of a non-pedal tricycle seems appropriate in stimulating physical activity in individuals living in nursing homes. Besides the motivation to move around, walking resistance and speed improved.
PUBLICATION DATA

Received 02092020

Accepted 09022021

Published 01062021
KEYWORDS: Older people | Gait speed | Gait distance | Intervention

\section{INTRODUCTION}

Aging is a complex and natural phenomenon that no one can avoid, and it is characterized by biological, physiological, and psychosocial alterations in all living beings. ${ }^{1}$ Those living in nursing homes usually present different needs and dependence degrees. Actually, older people with no family or those who are mistreated, suffer abuse or violence, or with no self-care capacity, more often live in nursing homes. ${ }^{2}$ For those living in nursing homes, physical dependence and the inability to perform daily living activities are more pronounced than their peers, mainly because of a higher incidence of illness, physical impairment, and psychological, social, and affective issues. ${ }^{3}$ Elderly individuals living in nursing homes are usually inactive and have less interaction with each other as they often spend their time watching TV or laying down around in their dorms, and this behavior might be due to the routine of most nursing homes. ${ }^{4}$

It has been reported that unstimulated elderly individuals, such as those living in nursing homes, are provided with fewer opportunities for moving around; consequently, they present increased muscle weakness, fall risk, gait impairment, depression, and cognitive decline. ${ }^{5,6}$ Therefore, it seems reasonable to develop strategies that could motivate inactive elderly adults to be active instead of sitting or laying down around on their beds for long 
periods. A strategy adopted in this study involved the use of a non-pedal tricycle, which is based on equipment used mainly in Europe, known as the "PETRA RaceRunning" tricycle. ${ }^{7}$ Overall, the adopted device contains a saddle, a support to lean on the trunk anteriorly, an open rear frame that allows movement of the lower limbs in different modes, one front, and two back wheels, and no pedals (the propulsion being generated by the foot contacting the ground). This device can maintain users' motivation and adherence to move around, independently of their fitness level. According to its inventor, the benefits of a non-pedal tricycle are promoting balance and locomotion, ${ }^{7}$ to avoid motor impairments. Through locomotion, the users of a non-pedal tricycle could improve muscle strength, balance, and resistance, which in turn improve their quality of life. ${ }^{8}$

Previous investigators have described how younger and older adults use the nonpedal tricycle as they move along a pathway. ${ }^{9}$ Overall, they found that both younger and older adults could perform the proposed task at the same speed, although older individuals presented more motion with the hip and less with the knee joints compared to younger ones. As older adults could transport themselves with a non-pedal tricycle, which seemed a safe way to exercise, it would be appropriate to test this in individuals living in nursing homes. To our knowledge, there is no information about the use of non-pedal tricycles by inactive elderly individuals. However, due to the general characteristics of individuals living in nursing homes, the goal of this study was twofold: to investigate the feasibility of a protocol using a non-pedal tricycle in older individuals living in nursing homes and its effects on improving endurance, walking speed, and functional mobility.

\section{METHODS}

\section{Participants}

A convenience sample of 24 individuals aged from 60 to 93 years (11 females, 13 males), who lived in two different public nursing homes in Caraguatatuba, SP, Brazil, was randomly allocated into two groups: control group (CG) and intervention group (IG). The inclusion criteria were as follows: understand simple verbal commands, walk with or without assistance, and present medical clearance for participation. The exclusion criteria were neurological, physical, and/or mental impairment that could compromise their participation in the study.

All procedures were performed with adequate understanding and written signed informed consent was obtained from all participants or their legal guardians. The institutional research ethics committee approved this study.

\section{Procedures}

All participants were instructed to keep their routine at their nursing homes during engagement in the study, which included medical and physical therapy, and nutritionist and psychologist attendances. Participants from IG, in addition to the routine, received practice sessions using a non-pedal tricycle to move around the nursing home, under the supervision of at least one experienced physical education instructor and a nurse from the nursing home. The intervention sessions were offered twice a week, with at least one day between sessions, 
for 12 weeks, summing a total of 24 sessions. Each session lasted up to $45 \mathrm{~min}$, with resting intervals provided according to the individual needs.

At the beginning of each practice session, the saddle and the wheels were adjusted according to the participant's height to accommodate her/him on the non-pedal tricycle (Figure 1). All participants wore comfortable clothes and shoes and were instructed to move at a self-selected speed and make turns as necessary around the area. One instructor was constantly next to the participant, providing verbal instructions and motivating them to move around.

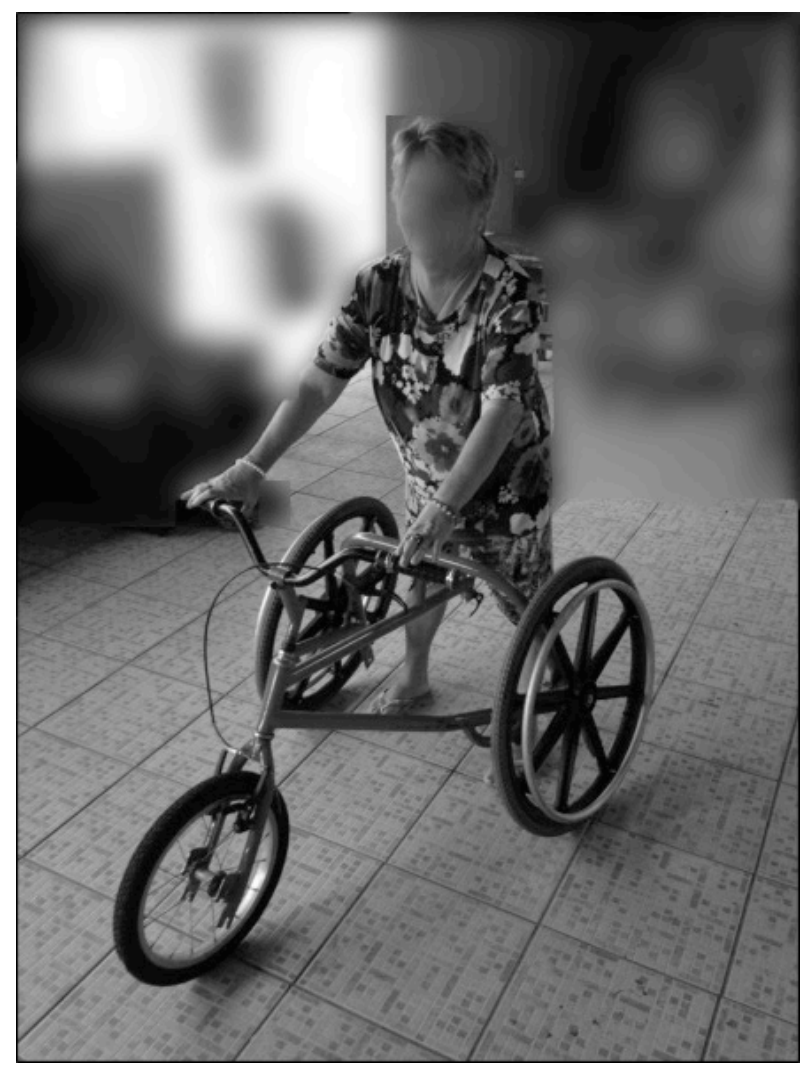

Figure 1. Illustration of a participant from the intervention group on the non-pedal tricycle.

During all practice sessions, the blood pressure of each participant was assessed by a nurse at the beginning and end of each session and after 10 min of rest as a safety precaution. The progression of the sessions was achieved by increasing the effective duration of movement with the non-pedal tricycle and decreasing the time for resting.

\section{Outcome measures}

All participants were assessed one week before the first training session and one week after the last training session provided to IG. One experienced researcher took the lead on all assessments and provided standardized instructions for each test to ensure consistency in test administration. Participants from both groups ( $C G$ and IG) underwent the 
following evaluations: 6-minute walk test, ${ }^{10} 10$-meter walk test, ${ }^{11}$ and timed-up and go test. ${ }^{12}$ Rest intervals were provided between tests according to individual needs.

Endurance was measured during the 6-minute walk test. Participants were instructed to walk at a comfortable speed along a 30-m-long pathway, turning around a cone at each end. The total distance covered during the 6-min period was recorded for each participant.

Gait speed was measured during the 10-meter walk test, as participants were required to walk $10 \mathrm{~m}$ at a comfortable speed. A stopwatch was used to measure the time required to cover the intermediate $6 \mathrm{~m}$, excluding the periods of acceleration and deceleration.

Finally, functional mobility was measured during the timed-up and go test, wherein the participant had to rise from a chair, walk $3 \mathrm{~m}$, turn around a cone, return to the chair, and sit down. One researcher tracked the time taken to complete the task using a stopwatch.

It is important to note that the researcher who did the randomization and data analysis was not involved in any assessment or training session. Conversely, complete blinding was not possible for the researcher who performed the intervention sessions and the assessment due to personnel constraints.

\section{Statistical analysis}

As normality (Shapiro-Wilk tests) and homogeneity (Levene tests) of variance were confirmed, parametric tests were employed. Four analyses of variance (ANOVAs) were employed using the groups ( $C G$ and IG) as a factor. The dependent variables for each test were age, body mass, height, and duration of stay in the nursing homes. Three two-way ANOVAs were employed using the groups and time of assessment (pre and post) as factors, the latter was treated as repeated measures. The dependent variables were distance walked (6-minute walk test), mean walking speed (10-meter walk test), and duration (timed-up and go test). When necessary, Tukey's post hoc tests were conducted. An alpha level of 0.05 was adopted for all statistical tests, which were conducted using Statistical Package for the Social Sciences software.

Within- and between-group effect sizes were calculated as the difference in mean values from each assessment divided by the pooled standard deviation. Effect sizes were defined using Cohen's classification ( $d=0.2$, small; $d=0.5$, medium; $d=0.8$, large). ${ }^{13}$

\section{RESULTS}

Ten participants were excluded from the final analysis due to Parkinson's disease $(n=2)$, Alzheimer's disease $(n=1)$, death $(n=2)$, incomplete assessment in one or more tests because of weakness $(n=3)$, and pain not related to their participation in the study $(n=2)$. Table 1 presents the general information of the remaining participants. ANOVA revealed no difference between groups for age $\left(F_{1,12}=0.01, p=0.91\right)$, body mass $\left(F_{1,12}=1.14, p=0.31\right)$, height $\left(F_{1,12}=0.32, p=0.58\right)$, and duration of stay in the nursing home $\left(F_{1,12}=0.19, p<0.66\right)$. 
Table 1 - General characteristics of participants from control and intervention groups related to age, body mass, height, and duration of stay in nursing home.

\begin{tabular}{|c|c|c|c|c|c|}
\hline ID & Sex & $\begin{array}{l}\text { Age } \\
\text { (yrs.) }\end{array}$ & $\begin{array}{c}\text { Mass } \\
(\mathrm{kg})\end{array}$ & $\begin{array}{l}\text { Height } \\
(\mathrm{m})\end{array}$ & $\begin{array}{c}\text { Nursing home } \\
\text { (yrs.) }\end{array}$ \\
\hline \multicolumn{6}{|c|}{ Control group } \\
\hline 1 & $\mathrm{~F}$ & 78 & 78.0 & 1.53 & 1 \\
\hline 2 & $\mathrm{~F}$ & 69 & 50.0 & 1.51 & 8 \\
\hline 3 & M & 66 & 59.0 & 1.70 & 3 \\
\hline 4 & M & 67 & 61.6 & 1.69 & 7 \\
\hline 5 & $F$ & 81 & 38.4 & 1.52 & 19 \\
\hline 6 & $\mathrm{~F}$ & 70 & 60.5 & 1.61 & 3 \\
\hline \multirow[b]{3}{*}{$S$} & $\mathrm{~F}$ & 93 & 49.6 & 1.50 & 45 \\
\hline & Mean & 74.6 & 56.7 & 1.58 & 12.3 \\
\hline & ard error & 3.3 & 4.4 & 0.03 & 5.9 \\
\hline \multicolumn{6}{|c|}{ Intervention group } \\
\hline 1 & M & 84 & 65.5 & 1.55 & 4 \\
\hline 2 & M & 72 & 77.3 & 1.51 & 3 \\
\hline 3 & M & 81 & 55.3 & 1.49 & 3 \\
\hline 4 & M & 69 & 60.2 & 1.60 & 5 \\
\hline 5 & $\mathrm{~F}$ & 64 & 48.4 & 1.46 & 23 \\
\hline 6 & M & 83 & 60.2 & 1.67 & 20 \\
\hline \multirow[b]{3}{*}{ St } & $\mathrm{F}$ & 74 & 76.3 & 1.61 & 7 \\
\hline & Mean & 75.2 & 63.3 & 1.56 & 9.3 \\
\hline & rd error & 3.3 & 4.4 & 0.03 & 3.2 \\
\hline
\end{tabular}

Figure 2 presents the results of each participant as well as the mean (standard error) for participants from the CG (left panel) and IG (right panel) at pre-and post-assessment. ANOVA for the distance covered during 6 min did not reveal a significant effect for both groups $\left(F_{1,12}=0.7, p=0.79\right)$ but revealed a significant effect for assessment $\left(F_{1,12}=6.31\right.$, $p=0.027, d=0.35)$ and interaction between groups and assessment $\left(F_{1,12}=10.81, p=0.007\right.$, $d=0.47$ ). Post hoc tests indicated no difference between both groups at pre-assessment and demonstrated that only participants from IG increased the distance at post-assessment $(d=0.45)$.

ANOVA for the mean walking speed did not reveal a significant effect for the groups $\left(F_{1,12}=0.77, p=0.39\right)$ and assessment $\left(F_{1,12}=3.28, p=0.09\right)$ but revealed an interaction between the groups and assessment $\left(F_{1,12}=9.01, p=0.011, d=0.43\right)$. Post hoc tests indicated no difference between both groups at pre-assessment and revealed that only participants from IG increased the mean walking speed at post-assessment $(d=0.75)$.

ANOVA for the duration to complete the "timed-up and go test" did not reveal significant effects for the groups $\left(F_{1,12}=4.11, p=0.06\right)$, assessment $\left(F_{1,12}=0.89, p=0.36\right)$, or interaction between the groups and assessment $\left(F_{1,12}=3.89, p=0.07\right)$. 

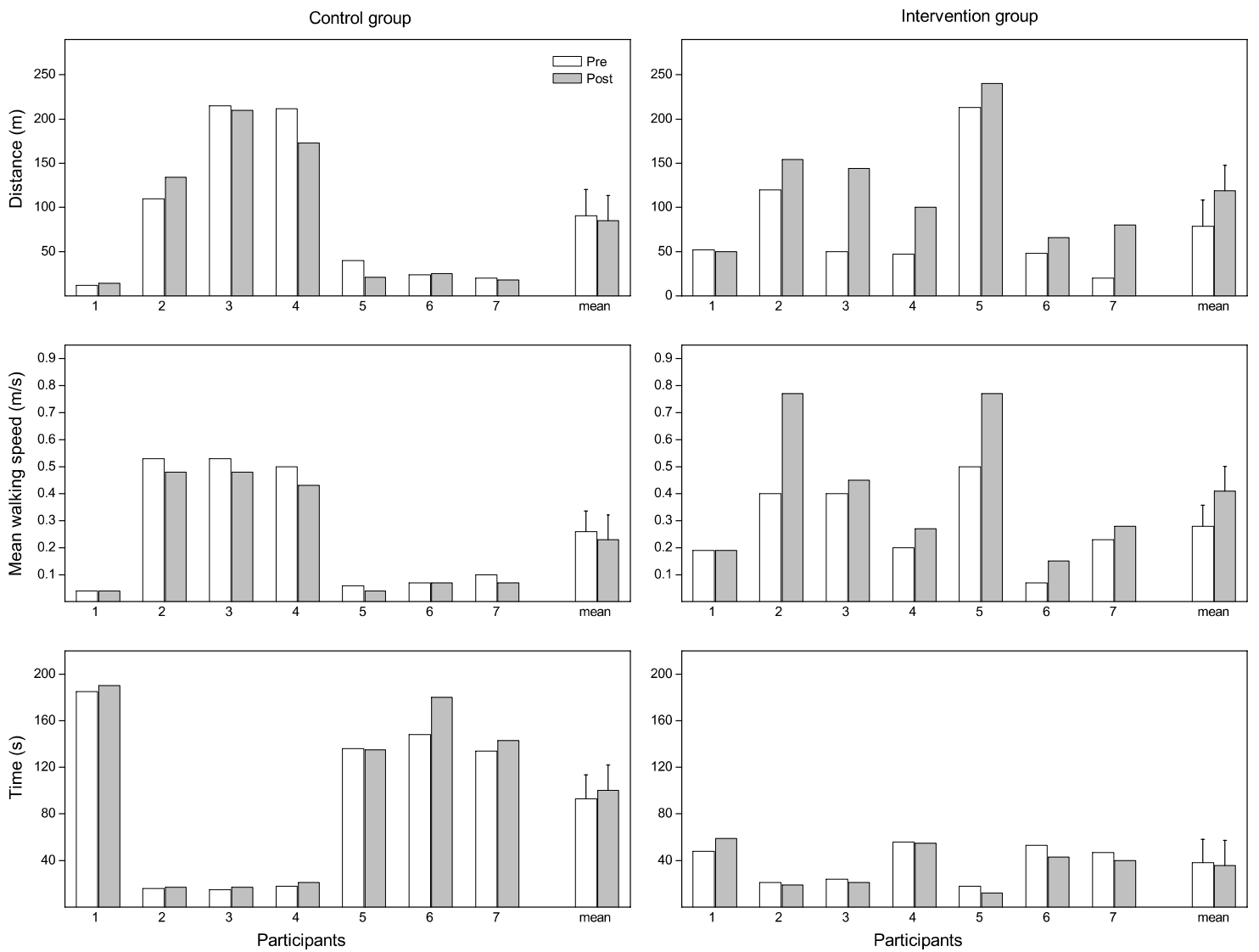

Figure 2. Individual and mean (standard error) values for distance (top), mean walking speed (middle), and time spent to conclude test (lower) of participants from the control (left panel) and intervention (right panel) groups at pre (white bars) and post (grey bars) assessments.

\section{DISCUSSION}

In this study, we investigated the feasibility of a protocol using a non-pedal tricycle in elderly individuals living in nursing homes and its effects in terms of improving endurance, walking speed, and functional mobility. In general, the results revealed that it is possible to employ a non-pedal tricycle in nursing homes, thus promoting the benefits regarding endurance and walking speed. Thus, the use of a non-pedal tricycle seems appropriate in stimulating physical activity in sedentary elderly individuals living in nursing homes.

Although IG displayed improved endurance and walking speed, it is important to note that their overall scores in the 6-minute walk and 10-meter walk tests were very low when compared to community-dwelling older individuals, ${ }^{14,15}$ or even to individuals with stroke before an intervention protocol. ${ }^{16}$ The reason for this can be due to the low levels of physical activity presented by nursing home residents ${ }^{17}$ and their marked diminished functional abilities. ${ }^{18}$ However, improvement in both tests by those who underwent the 
practice sessions can be attributed to the possibility of some physical activity engagement, which should be aimed at with the elderly population.

It is important to note that even though we failed to keep track of the effective time that participants moved with the non-pedal tricycle, most of them spent more time resting than moving around, although they stayed on the tricycle even during the resting intervals. This fact can justify why they showed no improvement in their duration to complete the timedup and go test, which involves standing up from a sitting position and walking with changes in the direction as fast as possible. We suggest that the way the practice sessions were conducted in this study enabled IG to improve balance but not the lower limb strength. However, we should be cautious with this suggestion as these aspects were not directly measured.

Although we did not employ any instrument for registering motivation and adherence to the use of a non-pedal tricycle, it is important to mention that even those individuals who resisted to use the equipment in the first sessions were interested and motivated afterward to move around the nursing home with it. An employee of one of the nursing homes reported that one elderly individual was motivated to leave his dorm only for the practice sessions. Another requested the use of the equipment to move to the opposite end of the nursing home to observe an instructor of another activity (something he would not do without the non-pedal tricycle). Based on these facts, it seems that the non-pedal tricycle, besides being able to promote locomotion in nursing home residents, stimulates physical activity and, most importantly, it is a tool that can be used even with those presenting gait impairments. Therefore, the use of a non-pedal tricycle might constitute a different and innovative way of promoting physical activity for those living in nursing homes.

To our knowledge, this is the first study investigating the viability of using a nonpedal tricycle to promote the physical activity of nursing home residents. Although this pilot study is relevant and presents important results, several limitations should be pointed out. The major limitation was the limited sample size recruited as only two public nursing homes took part in the study. Thus, this should be taken into consideration while assessing the results and generalizability of the findings, and future studies should include more participants from an increased number of nursing homes. We also did not monitor the exercise intensity and did not keep track of the effective time of practice for each session. We suggest future studies to use a heart rate monitor and a chronometer during practice sessions. All tests were performed at the same time for each assessment (pre and post) and in the same order for all participants. Even though we offered time for resting as necessary, it would be more appropriate to employ each of the three selected tests on three different days due to the frailty of these participants and in different orders to avoid possible bias. Lack of blinding to practicing sessions by the evaluator could also cause bias, although there were no specific expectations on which test was expected to lead to an improvement in results between both groups. Last but not least, the assessment should comprise different aspects other than locomotion, such as the supervision of physical activity level per day, adherence, and motivation to participate in the practice sessions, among others. It is thus important to plan a clinical trial, following all the recommendations to conduct it, by considering the limitations of the present study. 


\section{CONCLUSION}

In conclusion, the results of this study revealed that it is possible to employ a nonpedal tricycle for those residing in nursing homes, independent of their physical activity level. The use of this device twice a week as a way to promote physical activity contributed to improving the walking resistance and walking speed of frail individuals.

\section{REFERENCES}

1. Kerrigan DC, Xenopoulos-Oddsson A, Sullivan MJ, Lelas JJ, Riley PO. Effect of a hip flexor-stretching program on gait in the elderly. Arch Phys Med Rehabil. 2003;84:1-6.

2. Prefeitura SP. Serviços para Cidadão (Centros de acolhida especial) - Instituição de Longa Permanência para Idoso (ILPI), http://www.capital.sp.gov.br/cidadao/familia-eassistencia-social/centros-de-acolhida/centros-de-acolhida-especial/instituicao-de-longapermanencia-para-idoso-ilpi (accessed February 5th 2020).

3. Cuddy AJC, Norton MI, Fiske ST. This old stereotype: the pervasiveness and persistence of the elderly stereotype. J Soc Issues. 2005;61:267-285.

4. Bessa MEP, Silva MJ. Motivações para o ingresso dos idosos em instuições de longa permanência e processos adaptativos: um estudo de caso. Texto Contexto Enferm. 2008;17:258-265.

5. Franch-Ubia O. Changes in gait in the elderly. Rev Neurol. 2000;31:80-83.

6. Santos DM, Melo SIL, Carneiro LC, Andrade MC. Características da marcha de idosos considerando a atividade física e o sexo. Fisioter Mov. 2008;21:137-148.

7. Hansen C. PETRA RaceRunner, https://by-conniehansen.com/product/racerunner-petraracerunner/ (accessed August 4th 2020).

8. Rikli RE, Jone CJ. Senior fitness test manual. 2nd ed. Champaign, IL: Human Kinetics; 2013.

9. Calve T, Russo Junior DV, Barela AMF. Strategies adopted by younger and older adults while operating a non-pedal tricycle. Braz J Phys Ther. 2018;22:64-69. doi: 10.1016/j.bjpt.2017.10.008.

10. Laboratories ATSCOPSFCPF. ATS statement: guidelines for the six-minute walk test. Am J Respir Crit Care Med. 2002;166:111-117. doi: 10.1164/ajrccm.166.1.at1102.

11. Bohannon RW, Andrews AW, Thomas MW. Walking speed: reference values and correlates for older adults. J Orthop Sports Phys Ther. 1996;24:86-90. doi: 10.2519/jospt.1996.24.2.86. 
12. Podsiadlo D, Richardson S. The timed "Up \& Go": a test of basic functional mobility for frail elderly persons. J Am Geriatr Soc. 1991;39:142-148. doi: 10.1111/j.1532-5415.1991.tb01616.x.

13. Cohen J. A power primer. Psychol Bull. 1992;112:155-159.

14. Lobo A, Santos P, Carvalho J, Mota J. Relationship between intensity of physical activity and health-related quality of life in Portuguese institutionalized elderly. Geriatr Gerontol Int. 2008;8:284-290. doi: 10.1111/j.1447-0594.2008.00478.x.

15. Steffen TM, Hacker TA, Mollinger L. Age- and gender-related test performance in community-dwelling elderly people: Six-Minute Walk Test, Berg Balance Scale, Timed Up \& Go Test, and gait speeds. Phys Ther. 2002;82:128-137. doi: 10.1093/ptj/82.2.128.

16. Gama GL, Celestino ML, Barela JA, Forrester L, Whitall J, Barela AM. Effects of gait training with body weight support on a treadmill versus overground in individuals with stroke. Arch Phys Med Rehabil. 2017;98:748-745. doi: 10.1016/j.apmr.2016.11.022.

17. Marmeleira J, Ferreira S, Raimundo A. Physical activity and physical fitness of nursing home residents with cognitive impairment: A pilot study. Exp Gerontol. 2017;100:63-69. doi: 10.1016/j.exger.2017.10.025.

18. Rothera I, Jones R, Harwood R, Avery A, Waite J. Health status and assessed need for a cohort of older people admitted to nursing and residential homes. Age Ageing. 2003;32:303-309. doi: 10.1093/ageing/32.3.303.

Citation: Calve T, Barela AMF. The use of a non-pedal tricycle to promote physical activity in nursing home residents: a pilot study. BJMB. 2021. 15(2): 118-126.

Editors: Dr Fabio Augusto Barbieri - São Paulo State University (UNESP), Bauru, SP, Brazil; Dr José Angelo Barela São Paulo State University (UNESP), Rio Claro, SP, Brazil; Dr Natalia Madalena Rinaldi - Federal University of Espírito Santo (UFES), Vitória, ES, Brazil.

Copyright:@ 2021 Calve and Barela and BJMB. This is an open-access article distributed under the terms of the Creative Commons Attribution-Non Commercial-No Derivatives 4.0 International License which permits unrestricted use, distribution, and reproduction in any medium, provided the original author and source are credited.

Funding: This research did not receive any specific grant from funding agencies in the public, commercial, or not-forprofit sectors.

Competing interests: The authors have declared that no competing interests exist.

DOI: https://doi.org/10.20338/bjmb.v15i2.194 